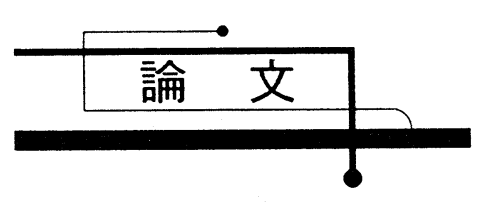

\title{
Computation on Turbulent Dilute Liquid-Particale Flows through a Centrifugal Impeller *
}

\author{
Yulin WU ** \\ Risaburo OBA $^{\dagger}$ \\ Toshiaki IKOHAGI $^{\dagger}$
}

\begin{abstract}
In the present work, the two-dimensional turbulent liquid-particle flow around a blade-toblade surface of a centrifugal impeller has been simulated by using a two-phase turbulence model, that is, the $\mathrm{k}-\varepsilon$ liquid turbulence model and the algebraic particulate phase flow turbulence model. The interaction between the liquid phase and the solid phase has been considered in the conservative momentum equations of the two-phase flow. Calculated results show that the turbulence model can predict essential features of the complex liquid-particle flow through the impeller.
\end{abstract}

Keywords : Liquid-Particle Flow, Turbulence Model, Numerical Analysis, Centrifugal Impeller

\section{INTRODUCTION}

Analyzing turbulent two-phase flows has attracted considerable attention due to its numerous industrial applications. Recent progress in modeling turbulent fluid-particulate flows was reported by Genchev \& Karpuzov (1980), Elghobashi \& AbouArab (1983), Pourahmadi \& Humphery (1983), Besnard \& Harlow (1985) and Chen \& Harlow (1985)[1]-[5]. Most of existing models are suitable only for relatively dilute mixtures, in which the particle-particle collisional effects and the fluctuation energy interaction between the liquid- and the particulate-phases are negligible. During the last decade, there has been an interesting development in modeling dense solid-liquid flows as shown in References of Ma \& Ahmadi (1988), Ahmadi \& Abu-Zaid (1990) and Asakura et al. (1991)[6]-[8].

In the present work, therefore, the two-dimensional turbulent liquid-particle flow around a bladeto-blade flow surface of a centrifugal impeller has been simulated by using two-phase turbulence model, that is, the k- $\varepsilon$ liquid turbulence model and the algebraic particulate phase turbulence model. In our numerical processing, a finite volume method is applied in a body-fitted coordinate system. Calculated results show that the turbulence model can predict essential features of the complex liquid-particle flow through a centrifugal impeller.

\section{GOVERNING EQUATIONS}

For incompressible liquid-particulate flow through a centrifugal impeller, the continuity-, the momentum- and the turbulent character-equations of both the liquid- and the particulate-phases in a twodimensional cartesian coordinate system, fixed on the impeller rotating with a speed $\omega$, can be written as follows:

\section{1 Liquid Phase}

(1) Continuity equation

$$
\left(\rho u_{j}\right)_{\mathbf{x}_{j}}=0
$$

* $\quad$ Received 12.5.1993

** Department of Hydraulic Engineering, Tsinghua University, Beijing, China

$\dagger \quad$ Institute of Fluid Science, Tohoku University, Sendai 980, Japan 
(2) Momentum equations

$$
\begin{aligned}
& \left\{\rho u_{i} u_{j}-\mu_{e}\left[\left(u_{j}\right)_{x_{i}}+\left(u_{i}\right)_{x_{j}}\right]\right\}_{x_{j}} \\
& =-P^{\prime}{ }_{x_{i}}+\frac{\rho_{p}}{\tau_{r p}}\left(u_{p i}-u_{i}\right)-2 \rho e_{i l k} \omega_{l} u_{k}
\end{aligned}
$$

where

$$
\begin{aligned}
& \tau_{r p}=\frac{\overline{\rho_{p}} d_{p}^{2}}{18 \mu}\left(1+R_{e p}^{2 / 3} / 6\right)^{-1}, \\
& R_{e p}=\left|\vec{u}-\vec{u}_{p}\right| d_{p} / v
\end{aligned}
$$

The subscript $x_{j}$ represents the derivative with respect to $x_{j}$.

The term - $2 \rho e_{i k l} \omega_{l} u_{k}$ represents the Coriolis force.

(3) Equation of turbulent kinetic energy $k$

$$
\left\{\rho k u_{j}-\frac{\mu_{e}}{\sigma_{e}} k_{x_{j}}\right\}_{x_{j}}=G_{k}-\rho \varepsilon
$$

where

$$
G_{k}=\mu_{t}\left[\left(u_{i}\right)_{x_{j}}+\left(u_{j}\right)_{x_{i}}\right]\left(u_{j}\right)_{x_{j}}
$$

(4) Equation of turbulent energy dissipation rate $\varepsilon$

$$
\begin{aligned}
& \left\{\rho \varepsilon u_{j}-\frac{\mu_{e}}{\sigma_{\varepsilon}} \varepsilon_{x_{j}}\right\}_{x_{j}} \\
& =C_{\varepsilon 1} \frac{\varepsilon}{k} G_{k}\left(1+C_{\varepsilon 3} R_{f}\right)-C_{\varepsilon 2} \rho \frac{\varepsilon^{2}}{k}
\end{aligned}
$$

where $R_{f}=k^{2} / \varepsilon^{2}\left(\omega / r^{2}\right)(\omega r)_{r}$ represents the effect of rotation on liquid turbulence.

(5) Boussinessq eddy viscosity $\mu_{t}$

$$
\mu_{t}=\rho C_{\mu} \frac{k^{2}}{\varepsilon}
$$

Constants in tha $k-\varepsilon$ turbulence model are taken as $C_{\mu}=0.09, \sigma_{k}=1.0, \sigma_{\varepsilon}=1.3, C_{\varepsilon 1}=1.44, C_{\varepsilon 2}=1.92, C_{\varepsilon 3}=0.8$

\section{2 Particulate Phase}

(1) Continuity equation

$$
\left[\rho_{p} u_{p j}-\frac{v_{p}}{\sigma_{p}}\left(\rho_{p}\right)_{x_{j}}\right]_{x_{j}}=0
$$

(2)Momentum equations

$$
\begin{aligned}
& \left\{\rho_{p} u_{p i} u_{p j}-\mu_{p}\left[\left(u_{p i}\right)_{x_{j}}+\left(u_{p j}\right)_{x_{j}}\right]\right\}_{x_{j}} \\
& =\frac{v_{p}}{\sigma_{p}}\left[u_{p i}\left(\rho_{p}\right)_{x_{j}}+u_{p j}\left(\rho_{p}\right)_{x_{i}}\right]_{x_{j}}+ \\
& +\frac{\rho_{p}}{\tau_{r p}}\left(u_{i}-u_{p i}\right)-2 \rho_{p} e_{i l k} \omega_{l} u_{p k}+0.5\left(\rho_{p} \omega^{2} r^{2}\right) x_{i}
\end{aligned}
$$

(3) Eddy viscosity of particulate phase $v_{p}$

The algebraic turbulence model of the particulate phase is used in this computation. So the eddy viscosity $v_{p}$ of the particulate phase can be expressed in the following Hinze-Tchen equation:

$$
\frac{v_{p}}{v_{t}}=\frac{k_{p}}{k}=\left(1+\frac{\tau_{r p}^{\prime}}{\tau_{t}}\right)^{-1}
$$

where the particle dynamic response time $\tau_{r p}^{\prime}$ and the fluctuation time of liquid turbulence $\tau_{t}$ are shown in the following form

$$
\begin{gathered}
\tau_{r p}^{\prime}=\frac{\bar{\rho}_{p} d_{p}^{2}}{18 \mu} \\
\tau_{t}=l / u^{\prime}=\sqrt{3 / 2} C_{\mu}^{3 / 2} \frac{k}{\varepsilon}
\end{gathered}
$$

\section{TRANSFORMATION OF THESE EQUATIONS OF MOTION}

In many flow problems, the boundary geometries are very complex, especially for internal flow problems with complicated boundaries, such as those of centrifugal impellers. So the use of nonorthogonal body-fitted coordinate (BFC) systems can be beneficial in many aspects. It is not only why the boundary geometries can be represented more closely using BFC systems, but also why the grid-refined solution can be easily obtained.

The governing equations of motion for the twodimensional relatively steady incompressible liquidparticle flow through such an impeller, expressed in the BFC systems $(\xi, \eta)$, can be represented by the following transport model equation (10) in the conservative form, in which $\phi$ denotes all the dependent variables respectively and $\Gamma$ is the diffusion coefficient:

$$
\begin{aligned}
& \left(U_{1} \phi\right)_{\xi}+\left(U_{2} \phi\right)_{\eta}-\left[\left(J \alpha_{1} \Gamma \phi_{\xi}\right)_{\xi}+\left(J \alpha_{2} \Gamma \phi_{\eta}\right)_{\eta}\right] \\
& =S_{d}+J S^{\phi}
\end{aligned}
$$

where $U_{1}$ and $U_{2}$ are contravariant velocity components in the body-fitted coordinates.

$$
U_{1}=J\left(\xi_{x} u+\xi_{y} v\right), U_{2}=J\left(\eta_{x} u+\eta_{y} v\right)
$$

$S^{\phi}$ is the source term indicated on the right side of Eqs.(1) to (4) and Eqs.(7) and (8) respectively.

$S_{d}$ is the extra source term resulting from non-orthogonality, that is,

$$
S_{d}=\left(J \alpha_{4} \Gamma \phi_{\eta}\right)_{\xi^{+}}+\left(J \alpha_{4} \Gamma \phi_{\xi}\right)_{\eta}
$$

where $\alpha_{1}, \alpha_{2}$ and $\alpha_{4}$ are transformation coefficients. 
Table $1 \phi, \Gamma$ and $S^{\phi}$ appearing in Eq. (10)

\begin{tabular}{|l|c|c|c|}
\hline liquid phase & $\phi$ & $\Gamma$ & $S^{\phi}$ \\
\hline (1) continuity & $\rho$ & & \\
\hline (2) momentum & $\rho u_{i}$ & $\mu_{e}$ & $-p_{x i}^{\prime}-\rho_{p}\left(u_{p i}-u_{i}\right) / \tau_{r p}-2 \rho e_{i k k} \omega_{i} u_{k}$ \\
\hline (3) $k$ Eq. & $\rho k$ & $\mu_{e} / \sigma_{k}$ & $G_{k}-\rho \varepsilon$ \\
\hline (4) $\varepsilon$ Eq. & $\rho \varepsilon$ & $\mu_{e} / \sigma_{\varepsilon}$ & $C_{\varepsilon 1} \varepsilon / k G_{k}\left(1+C_{\varepsilon 3} R_{f}\right)-C_{\varepsilon 2} \rho \varepsilon^{2} / k$ \\
\hline particle phase & & & \\
\hline (1) continuity & $\rho_{p}$ & $v_{p} / \sigma_{p}$ & $v_{p} / \sigma_{p}\left[u_{p i}\left(\rho_{p}\right)_{x j}+u_{p j}\left(\rho_{p}\right)_{x i}\right]_{x i}+\rho_{p}\left(u_{i}-u_{p i}\right) / \tau_{r p}$ \\
\hline (2) momentum & $\rho_{p} u_{p i}$ & $\mu_{p}$ & $-2 \rho_{p} e_{i l k} \omega_{i} u_{p k}+0.5 \omega^{2} r^{2} \rho_{p}$ \\
\hline
\end{tabular}

$$
\begin{aligned}
& \alpha_{1}=\xi_{x}^{2}+\xi_{y}^{2} \quad, \alpha_{2}=\eta_{x}^{2}+\eta_{y}^{2} \\
& \alpha_{4}=\xi_{x} \eta_{x}+\xi_{y} \eta_{y}
\end{aligned}
$$

The terms of variable $\phi$ and coefficients $\Gamma$, as well as the source terms $S^{\phi}$ are given in Table 1.

\section{SOLUTION AND FINITE DIFFERENCE FORMULATION}

\section{1 Discretization of Equations}

Discretization of Eq. (10) is performed by using finite difference approximations in the transformed domain. The second order central differencing is used for approximating the diffusion and the source terms. For the convective terms, the hybrid differencing scheme (Spalding, 1972)[9] is employed (i. e. using central differencing for cell Peclet number less than or equal to 2 and switching to upwind differencing when the cell Peclet number is greater than 2).

The finite difference equation is arranged by collecting terms according to the grid nodes around a central volume as shown in Fig.1. The final expression is given by Eq. (14) in which A represents the link coefficients between the grid nodes B, E, W, N, S

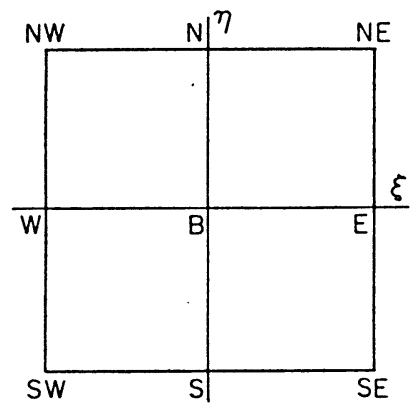

Fig. 1 Two-dimensional grid structure and labeling around a grid node $B$. and NE, NW, SE, and SW.

$$
A_{B} \phi_{B}=A_{E} \phi_{E}+A_{W} \phi_{W}+A_{N} \phi_{N}+A_{S} \phi_{S}+S_{\imath}
$$

where

$$
\begin{aligned}
& S_{\imath}=S+A_{N E} \phi_{N E}+A_{N W} \phi_{N W}+A_{S E} \phi_{S E}+A_{S W} \phi_{S W} \\
& S=S_{d}+J S^{\phi} \\
& A_{B}=A_{E}+A_{W}+A_{N}+A_{S}
\end{aligned}
$$

\section{2 Solution Procedures of Two-Phase Flows}

The present numerical method for solving liquidparticulate flows contains the following steps:

(1) Guess the initial velocity and the pressure fields of a single liquid phase flow in the calculated domain.

(2) Solve for the velocity, the pressure and the turbulent characters $\mathrm{k}$ and $\varepsilon$ in the single flows, by using the SIMPLEC method (proposed by Spalding 1980, and improved by Chen Y.S. 1986)[10],[11] to get a coarse convergence solution of the flows.

(3) Based on the single flow solution, we solve for the particulate turbulent flow field, by using the algebraic turbulence model.

(4) Calculate the interaction terms between the liquidand the particulate-phases to get some sourceterms in control equations for the liquid flow.

(5) Solve again the momentum equations, the turbulent energy and its dissipation rate equations for the single flow but including the interaction terms.

(6) Set the new variables as improved ones and return to the step (3); repeat the process untill it converges.

\section{3 SIMPLEC Solver for Incompressible Flows}

The governing equation solving the incompressible turbulent flows are nonlinear and are strongly coupled. Iterative procedures are employed to drive the equations to converge the solutions. It is particu- 
larly important for such an incompressible flow to satisfy the continuity equation and the momentum equations at the same time. A velocity-pressure correction procedure is used in the present study to drive the pressure field and velocity field to be divergence free. It requires a staggering grid system, in which the velocity components are solved and are stored at the grid nodes, and the pressure $p$ is located at the corners of the control volume of velocities. In this way, the coupling velocities and the pressure can be also enforced.

In the SIMPLEC procedure, the velocity corrections $u_{B}^{\prime}$ and $v_{B}^{\prime}$ can be calculated by the following equations:

$$
\begin{aligned}
& u_{B}^{\prime}=-\left[1 /\left(A_{B}^{u}-\sum A_{i}^{u}\right)\right] p_{x}{ }_{x}=-D_{u} p^{\prime \prime}{ }_{x} \\
& v_{B}^{\prime}=-\left[1 /\left(A_{B}^{v}-\sum A_{i}^{v}\right)\right] p_{y}^{\prime \prime}=-D_{v} p^{\prime \prime}{ }_{y}
\end{aligned}
$$

And the pressure correction value $p$ " can be obtained through solving a Poisson's equation (16) with the source term equal to the local divergence of the flow field $\left(u^{*}, v^{*}\right)$

$$
\left(D_{u} p_{x}^{\prime \prime}\right)_{x}+\left(D_{v} p_{y}^{\prime \prime}\right)_{y}=u_{x}^{*}+v_{y}^{*}
$$

\section{BOUNDARY CONDITIONS}

\section{1 Flow in Liquid Phase}

(1) Inlet boundary The inlet values of the radial velocity component $u_{r 1}$ and the circumferential one $u_{\theta 1}=\omega_{r 1}$ are directly specified and are easily altered. The inlet value of turbulent kinetic energy $k_{i n}$ adopts $0.5-1.5 \%$ of the inlet mean kinetic energy of flow, that is,

$$
\begin{aligned}
& k_{\text {in }}=0.005 u_{\mathrm{r} 1}^{2} \\
& \mu_{\text {in }}=\rho u_{r 1} l / 100
\end{aligned}
$$

where $l$ is the specific length of the inlet boundary, and

$$
\varepsilon_{i n}=C_{\mu} k_{i n}^{3 / 2} / l
$$

The values of $k_{i n}, \mu_{\text {in }}$ and $\varepsilon_{i n}$ do not severely affect on the final results.

(2) Outlet boundary The velocity components at the outlet of the calculated domain are deduced from their immediately upstream values by adding a fixed amount, whose amount is calculated to ensure overall mass conservation.

The outlet values of $k$ and $\varepsilon$ are unimportant, because limited effects of the upstream are known at a high Reynolds number and zero gradient is specified.

(3) Solid walls In the near wall regions, to avoid the need for detailed calculations the wall functions are introduced and are used in the finite difference calculations. That is, the total tangential velocity $V_{B}$ at the point B near the wall is corrected by the velocity profile

$$
V_{B}^{+}=(1 / \lambda) \ln \left(E Y_{B}^{+}\right)
$$

where $\lambda=0.41$ and $E=9.0$. And

$$
\begin{aligned}
& V_{B}^{+}=V_{B} / u_{\tau} \\
& u_{\tau}=\sqrt{\tau_{w} / \rho} \\
& Y_{B}^{+}=Y_{B} u_{\tau} / v
\end{aligned}
$$

where $Y_{B}$ is the normal distance from the point $B$ to the wall. $\tau_{B}$ is an approximation for the wall stress $\tau_{w}$ near the wall and is formulated by neglecting the convection and the diffusion of the turbulent kinetic energy equation in this region.

$$
\tau_{B}=C_{\mu}^{1 / 2} \rho k_{B}
$$

Then, we obtains

$$
\tau_{w}=U_{B} k_{B}^{1 / 2} \lambda / \ln \left(E Y_{B}^{+}\right)
$$

From the foregoing equation, $k_{B} / \tau_{B}=\left(C_{\mu}\right)^{-1 / 2} / \rho=$ constant, which implies that the wall flux of $k$ is zero. Accordingly zero normal gradient prescription for $k$ is appropriate. For the turbulent energy dissipation rate near the wall, the length scale is assumed to be proportional to the normal distance from the wall, which leads to

$$
\varepsilon=\left(C_{\mu} k_{B}\right)^{3 / 2} /\left(\lambda Y_{B}\right)
$$

(4) Boundary condition of pressure The correction values $p^{\prime}$ of SIMPLE scheme possess zero gradient specification everywhere except the inlet. Just one internal pressure $p$ specification at a point is then required to all the pressure to be calculated.

\section{2 Particulate Phase}

Variables in the particulate phase also possess zero normal gradient specification everywhere except the inlet. At the inlet particle velocity components are assumed to have the same values as those in the liquid flow, because there are not any experimental data on their inlet velocity difference. Even if the particle inlet velocity is taken as a slightly smaller value than that of liquid phase, the calculated results may still indicate that the particle outlet velocity is larger than the liquid one, which has been verified by experiments in Refs. 
[4], [10]. And the bulk density in the particulate phase at the inlet can be easily determined according to the known volumetric concentration.

\section{CALCULATED RESUlTS}

In the present work, because the flow passage in the impeller and its blade shape are basically two-dimensional, which is an actual model of a centrifugal slurry pump impeller (Wu, et al. 1993)[12], so that the two-dimensional turbulent dilute liquid-particle flow calculations have been carried out for this impeller. Its geometrical parameters are as follows:

impeller inlet diameter $D_{1}=130 \mathrm{~mm}$,

impeller outlet diameter $D_{2}=330 \mathrm{~mm}$,

rotating speed $n=1450 \mathrm{rpm}$,

radial inlet velocity $u_{r 1}=6 \mathrm{~m} / \mathrm{s}$,

particle diameter $d_{p}=1.0 \mathrm{~mm}$,

particle density $\rho=2.65 \mathrm{t} / \mathrm{m}^{3}$.

Fig. 2 to Fig. 5 respectively show the relative velocity vectors, its contour lines and the contour lines of pressure (maximum value $4.99 \times 10^{5} \mathrm{~Pa}$ ) and the turbulent kinetic energy at the single water phase flow condition. Fig. 6 to Fig. 9 show the same results of water flow for the two-phase flow condition with the particle volumetric concentration of $C_{v}=5 \%$. Fig. 10 and Fig. 11 show the relative velocity vectors and its contour lines of the particle phase at the same $C_{\nu}$ condition. Fig. 12 and Fig. 13 illustrate the same particle phase results as those in Fig. 10 and Fig. 11 for the $C_{\nu}=10 \%$ condition, as well as Fig. 14 and Fig. 15 when $C_{\nu}=2 \%$.

From these calculated results, the following remarks can be obtained.

(1) According to the comparison between Fig. 2

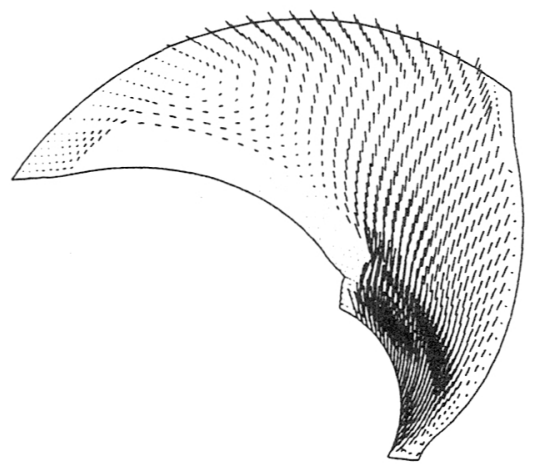

Fig. 2 Relative velocity field of single water phase flow $\left(n=1450 \mathrm{rpm}, u_{r 1}=6 \mathrm{~m} / \mathrm{s}\right)$. and Fig. 6, Fig. 3 and Fig. 7, existence of solid particles in the impeller has a little influence on the liquid flow at the dilute concentration $\left(C_{\nu}<10 \%\right)$. In the liquid flow in the impeller (Fig. 2 and Fig. 6), circulation occurs near the pressure surface of the blade from the mid span to the outlet resulting from large expansion of the flow passage sections as the blades are only four and as the attack angle is largely positive at the blade inlet at this operating condition.

But the existence of particles affects to the pressure developed by the impeller. The denser the particle concentration is in the impeller, the less the pressure of liquid flow field is at the impeller outlet.

(2) The velocity field in the particulate phase is different from that of the liquid phase (Fig .10 to Fig. 15). The velocity difference between these two phases takes place mainly near the outlet. That is, the relative particle velocity components at the outlet are larger than those in the liquid flow. The calculated results agree with the measured results by using LDV by Carder, et al.(1991)[13]. The results also agree with the observation of individual particle movement by using high-speed photography by Herbich and Christopher (1963)[14]. That is, the particles are not appreciably slowed down even at the large cross-sectional area of the impeller discharge ring, and their absolute tangential velocities do not increase towards the tangential velocities of transporting liquid.

(3) An increase in the volumetric particle concentration (Figs. 11 and 13) results in a marked difference between the particle velocities and the liquid ones, because the particulate phase with a large concentration $C_{v}$ as a pseudo-fluid in the present model keeps more inertia from the impeller inlet to the dis-

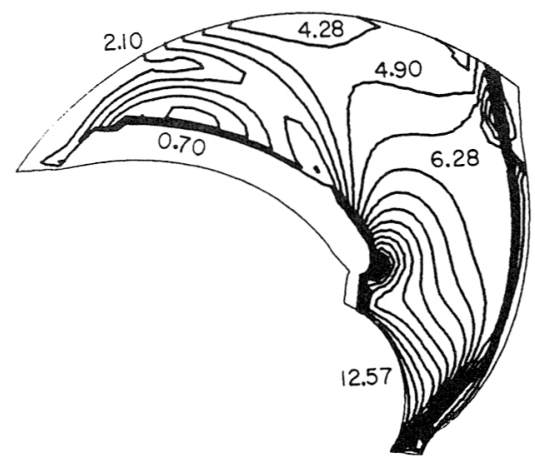

Fig. 3 Velocity contours of single water phase flow $\left(W_{\max }=13.27 \mathrm{~m} / \mathrm{s}\right)$. 


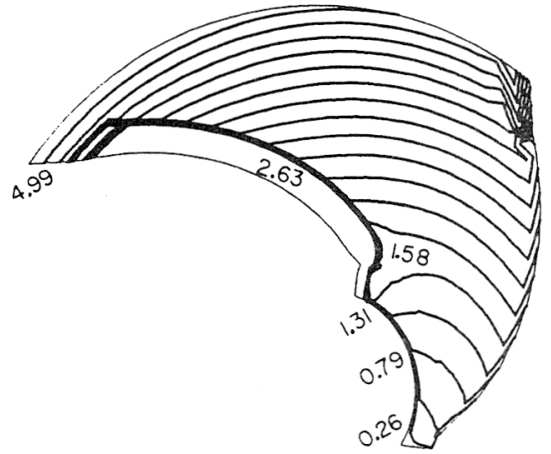

Fig. 4 Pressure contours of single water phase $\left(p_{\max }=4.99 \times 10^{5} \mathrm{~Pa}\right)$.

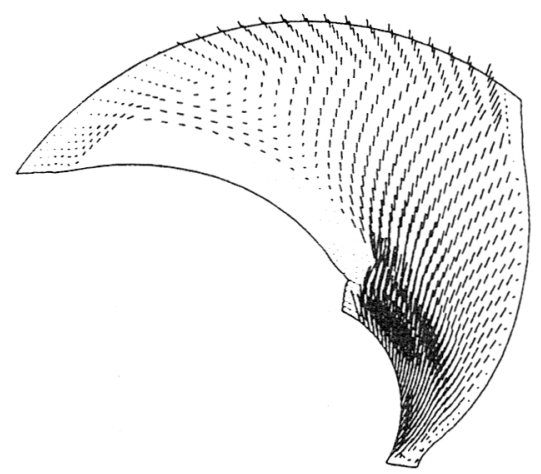

Fig. 6 Water velocity of two-phase flow $\left(C_{\nu}=5 \%\right)$.

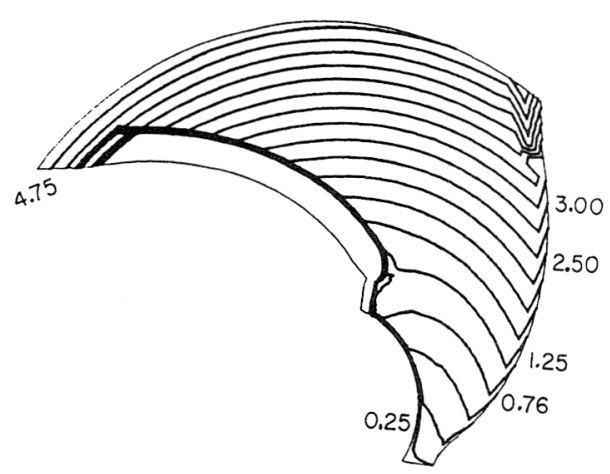

Fig. 8 Pressure contours of two-phase flow $\left(p_{\max }=4.75 \times 10^{5} \mathrm{~Pa}\right)$.

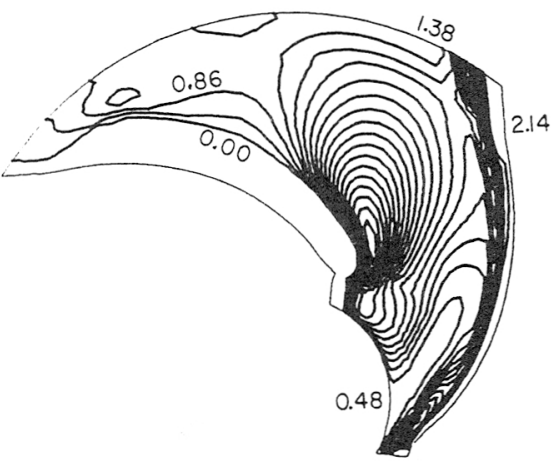

Fig. 5 Turbulent energy contours of single water flow $\left(k_{\max }=8.13 \mathrm{~m}^{2} / \mathrm{s}^{2}\right)$.

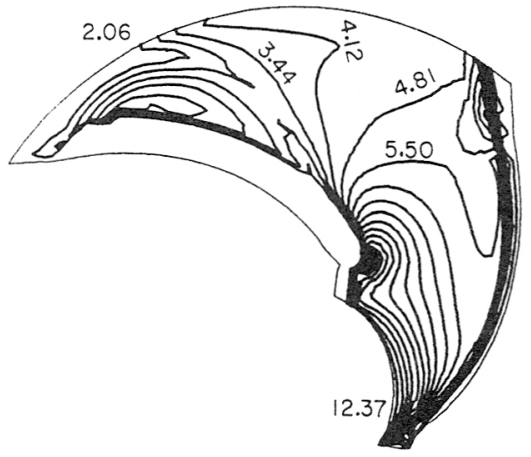

Fig. 7 Water velocity contours of two-phase flow $\left(W_{\max }=13.06 \mathrm{~m} / \mathrm{s}\right)$.

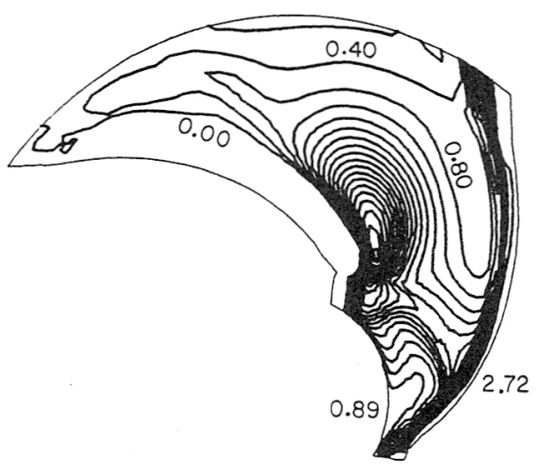

Fig. 9 Turbulent energy of two-phase flow $\left(k_{\max }=7.39 \mathrm{~m}^{2} / \mathrm{s}^{2}\right)$. 


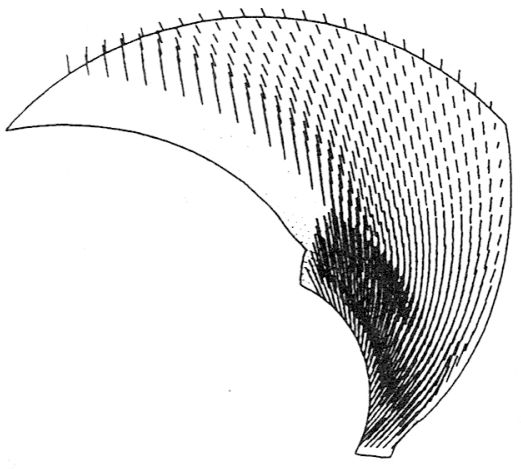

Fig. 10 Velocity field of particle $\left(C_{v}=5 \%\right)$.

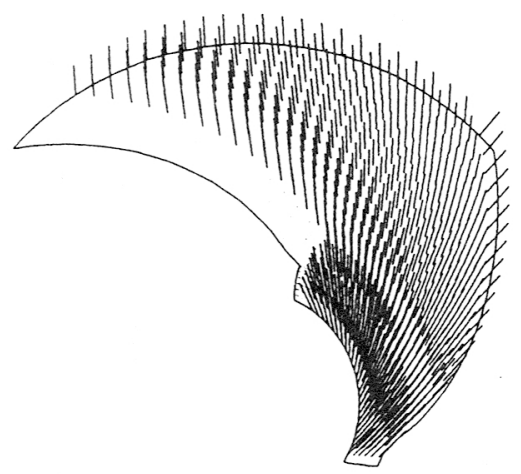

Fig. 12 Velocity field of particle $\left(C_{\mathrm{v}}=10 \%\right)$.

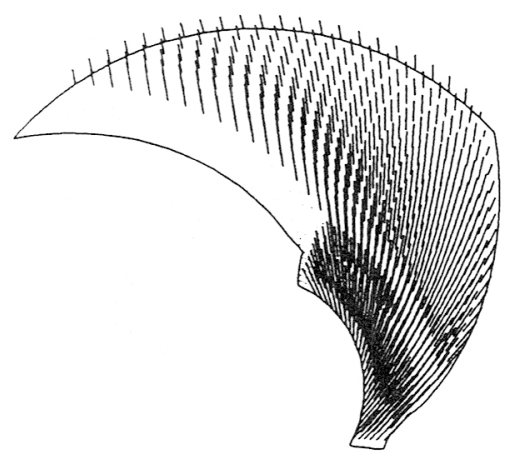

Fig. 14 Velocity field of particle $\left(C_{\nu}=2 \%\right)$.

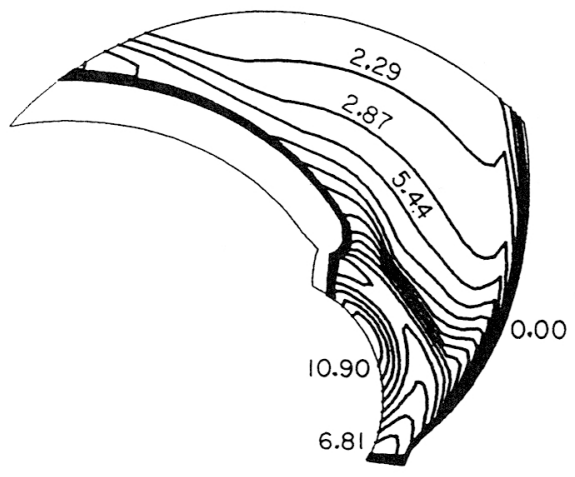

Fig. 11 Velocity of particle $\left(W_{\max }=10.9 \mathrm{~m} / \mathrm{s}\right)$.

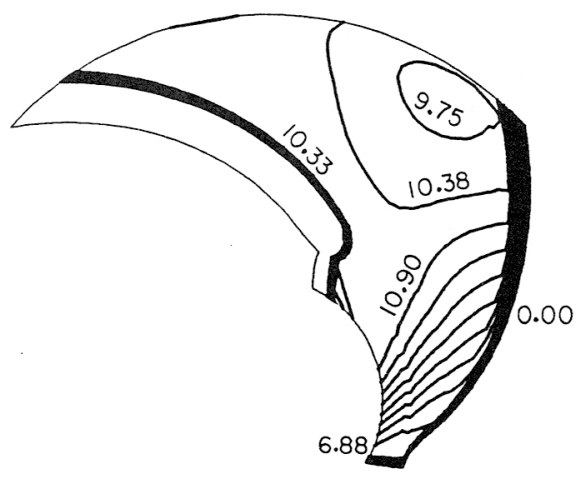

Fig. 13 Velocity of particle $\left(W_{\max }=10.9 \mathrm{~m} / \mathrm{s}\right)$.

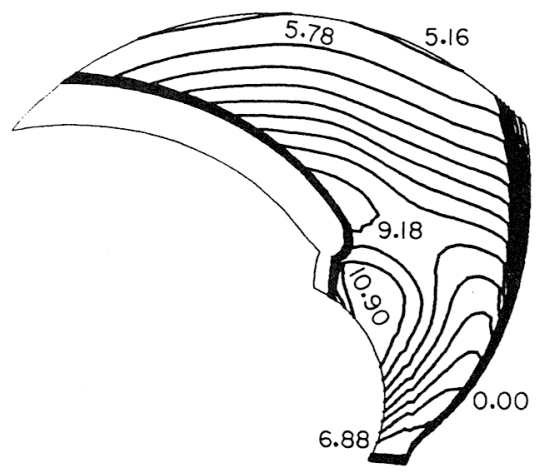

Fig. 15 Velocity of particle $\left(W_{\max }=10.9 \mathrm{~m} / \mathrm{s}\right)$. 
charge ring. With an increase in particle concentration, the particle velocities along the blade pressure surface will be higher than those along the suction surface at the outlet ring. This phenomenon also agrees with the observation of moving individual particles in centrifugal impellers by Wu, et al. (1993)[12], and suggests the existence of distorted particulate distributions in the impeller as shown by Roco and Reinhart (1980)[15].

\section{CONCLUSIONS}

(1) The turbulent liquid-particle flow model used in this paper can predict essential features of this flow in the centrifugal impellers at dilute concentrations.

(2) The velocity field of particulate phase is different from that of the liquid phase in the centrifugal impellers, especially near the outlet ring of the impeller. Relative velocities of particles are not slowed down near the outlet.

(3) At dilute concentrations, existence of particles scarcely affects the liquid-phase flow in the impeller. But it makes the pressure at the impeller oulet lower than that of the single phase flow a little. The denser the particle concentration is, the more the impeller outlet pressure decreases.

\section{NOMENCLATURE}

$\begin{array}{llr}A & : \text { link coefficients } & {[-]} \\ C & : \text { constants } & {[-]} \\ C_{v} & : \text { volumetric concentration } & {[-]} \\ d_{p} & : \text { diameter of particle } & {[\mathrm{m}]} \\ e_{i j k} & : \text { levy-civita symbol } & {[-]} \\ G & : \text { general generation } & {[-]} \\ J & : \text { Jacobian of transformation } & {[-]} \\ k & : \text { turbulent kinetic energy of liquid } & \\ & \text { phase } & {\left[\mathrm{m}^{2} / \mathrm{s}^{2}\right]} \\ k_{p} & : \text { turbulent kinetic energy of particulate } & \\ & \text { phase } & {\left[\mathrm{m}^{2} / \mathrm{s}^{2}\right]} \\ p & : \text { pressure } & {[\mathrm{Pa}]} \\ p^{\prime} & : \text { : } p-0.5 \rho \omega^{2} r^{2} & {[\mathrm{~Pa}]} \\ p^{\prime \prime} & : \text { correction value of pressure } p & {[\mathrm{~Pa}]} \\ r & : \text { radius } & {[\mathrm{m}]} \\ S_{d} & : \text { general additional source term } & {[-]} \\ S^{\phi} & : \text { general source term } & {[-]} \\ u, v \text { or } u_{j} & : \text { Reynolds mean velocity components in } & \\ & \text { cartesian coordinates } & {[\mathrm{m} / \mathrm{s}]} \\ u^{\prime}, v^{\prime} & : \text { correction values of velocities } u, v & {[\mathrm{~m} / \mathrm{s}]} \\ U_{1}, U_{2} & : \text { contravariant velocities in body-fitted } & \\ & \text { coordinates } & {[\mathrm{m} / \mathrm{s}]} \\ w & : \text { velocity produced by rotation } & {[\mathrm{m} / \mathrm{s}]}\end{array}$

$x_{j} \quad:$ cartesian coordinates

[m]

\section{GREEK LETTERS}

$\varepsilon \quad:$ rate of turbulent energy dissipation $\quad\left[\mathrm{m}^{2} / \mathrm{s}^{3}\right]$

$\alpha \quad$ : transformation coefficients

$\Gamma \quad:$ general diffusion coefficient $\quad[-]$

$\mu(v) \quad:$ liquid viscosity [Pa.s]

$\mu_{e}\left(v_{e}\right)$ : effective liquid viscosity [Pa.s]

$\mu_{\mathrm{e}}=\mu_{\mathrm{t}}+\mu$
$\mu_{t}\left(v_{t}\right) \quad:$ eddy viscosity of liquid $\quad$ [Pa.s]

$\mu_{p}\left(v_{p}\right)$ : eddy viscosity of particulate phase [Pa.s]

$\xi, \eta \quad:$ body-fitted coordinates [m]

$\rho \quad:$ density of liquid $\quad\left[\mathrm{kg} / \mathrm{m}^{3}\right]$

$\rho_{p} \quad:$ bulk density of particulate phase $\quad\left[\mathrm{kg} / \mathrm{m}^{3}\right]$

$\bar{\rho}_{p} \quad:$ material density of particle $\quad\left[\mathrm{kg} / \mathrm{m}^{3}\right]$

$\sigma_{\mu} \quad$ : constant [-]

$\sigma_{p} \quad:$ constant $\quad[-]$

$\tau^{p} \quad:$ fluctuation time of liquid turbulence [s]

$\tau_{t}^{\prime} \quad:$ dynamic response time of particle $\quad[\mathrm{s}]$

$\phi \quad$ : general variable $\quad[-]$

$\omega \quad$ : rotating speed $\quad[\mathrm{rad} / \mathrm{s}]$

\section{REFERENCES}

[ 1 ] Genchev, Z. D. and Karpuzov, D. S., J. Fluid Mech., Vol.101, 823-842 (1980).

[ 2 ] Elghobashi,S.E. and Abou-Arab,T.W., Phys. Fluids, Vol.26,931-938 (1983).

[ 3 ] Pourahmadi,F. and Humphery, J. A. C., Physco Chemical Hydrodynamics, Vol.4, 191-219 (1983).

[ 4 ] Besnard, D. C. and Harlow, F. H., Los Alamos National Laboratory, Report No. LA-10187MS (1985).

[ 5 ] Chen, C. P. and Harlow, F. H., Canadian J. Chem. Engng., Vol.63, 349-357 (1985).

[ 6 ] Ma,D.N. and Ahmadi,G., Powder Tech., Vol.56, 191219 (1988).

[7] Ahmadi,G. and Abou-Zaid,S., Int.J. Non-Newtonian Fluid Mechanics, Vol.35, 15-35 (1990).

[ 8 ] Asakura,K. et al., ASME FED, Vol.118, Liquid-Solid Flows, 45-57 (1991).

[ 9] Spalding,D.B., Int.J. Num. Methods Eng., Vol.4, 551562 (1972).

[10] Spalding,D.B., Imperial College, London, HTS/80/1 (1980).

[11] Chen,Y.S., NASA CR-178818 (1986).

[12] Wu,Y.L. et al., Proc. of Int. Symp. on Aeropace and Fluid Science, Sendai, 379-384 (1993).

[13] Cader,T. et al., ASME FED, Vol.118, Liquid-Solid Flows, 101 (1991).

[14] Herbich, J.B. and Christopher, R.J., Proc. of the IAHR Congress, London, 89-98 (1963).

[15] Roco,M. and Reinhart, E., BHRA, Hydrotransport, Vol.7, 359-376 (1980). 